\title{
LIGHT CURVE MODELS FOR SN 1987A AND DIAGNOSIS OF SUPERNOVA INTERIOR
}

\author{
Ken'ichi Nomoto ${ }^{1}$, Toshikazu Shigeyama ${ }^{1}$, and Masa-aki Hashimoto ${ }^{2}$ \\ ${ }^{1}$ Department of Earth Science and Astronomy, University of Tokyo, Tokyo \\ ${ }^{2}$ Max-Planck-Institut fur Astrophysik, Garching
}

\begin{abstract}
Presupernova evolution of the progenitor of SN 1987A, hydrodynamics of explosion (shock propagation, explosive nucleosynthesis), optical light curve due to shock heating and ${ }^{56} \mathrm{Co}$ decay, and X-ray and $\gamma$-ray light curves are calculated and compared with the observations of SN 1987A. Constraints on the mass of the hydrogen-rich envelope $M_{\text {env }}$ (i.e., mass loss history) and the helium abundance in the envelope are obtained from the progenitor's blue-red-blue evolution as well as from the light curve. The explosion energy $E$ and the mass and distribution of ${ }^{56} \mathrm{Ni}$ are inferred from the light curves. Models and observations are in reasonable agreement for $E / M_{\text {env }}=1.5 \pm$ $0.5 \times 10^{50} \mathrm{erg} / M_{\odot}, M_{\mathrm{env}}=5-10 M_{\odot}$, and $M_{\mathrm{Ni}} \sim 0.07 M_{\odot}$. Mixing of ${ }^{56} \mathrm{Ni}$ into the envelope is indicated.

Light curves of exploding bare helium stars are also calculated to see whether the observed Type Ib supernova light curves can be accounted for.

\section{INTRODUCTION}

SN $1987 \mathrm{~A}$ in the LMC is directly related to the three issues discussed at our Colloquium: chemical peculiarity, mass loss, and explosion. This supernova is providing us with valuable information on the three major uncertainties involved in the current theory of massive star evolution and explosion, namely, 1) the mechanism that tranforms collapse into explosion, 2) mass loss, and 3) convection (material mixing, in general). Because of the lack of clear understanding of these processes, the explosion energy $E$, mass and distribution of ${ }^{56} \mathrm{Ni}$, and the mass of the hydrogen-rich envelope $M_{\text {env }}$ in SN 1987 A are highly uncertain.

From the comparison between observations and the theoretical models of stellar evolution, explosion, and the optical, X-ray, $\gamma$-ray light curves, we will examine:

1) why was the progenitor of SN $1987 \mathrm{~A}$ blue,

2) how much $M_{\text {env }}$ was retained in the progenitor after mass loss,
\end{abstract}


3) how large is $E$,

4) what kind of heavy elements are synthesized and how they are distributed in the ejecta.

In addition to SN 1987A, explosion of bare helium stars is calculated to examine whether and under what conditions Type Ib-like light curves are realized.

These consideration will lead us deeper understanding of the mixing, mass loss, and explosion during the evolution of massive stars.

\section{WHY WAS THE PROGENITOR BLUE ?}

The most likely progenitor of SN $1987 \mathrm{~A}, \mathrm{Sk}-69202$, was surprisingly a B3 blue supergiant. Its luminosity is about $1.3 \times 10^{5} \quad L_{\odot}$ which corresponds to the presupernova luminosity of a helium core of $M_{\text {core }} \sim 6 M_{\odot}$ (Woosley 1988; Nomoto, Shigeyama, and Hashimoto 1987; Nomoto and Hashimoto 1988). Its main-sequence mass is $M_{\mathrm{ms}}=17-22 M_{\odot}$. From the luminosity and the spectral type, the radius of Sk-69202 is estimated to be about $3 \times 10^{12} \mathrm{~cm}$.

The occurrence of a Type II supernova from such a blue supergiant progenitor is not known before. It is very likely that the progenitor of SN $1987 \mathrm{~A}$ evolved first to become a red supergiant and then came back to the blue as indicated by the following observations. The UV observations have shown the existence of a circumstellar shell which is nitrogen overabundant and whose expansion velocity is less than $30 \mathrm{~km} \mathrm{~s}^{-1}$ (Panagia et al. 1987). The existence of a dust shell is also inferred from the IR observations (Chalavaev et al. 1987). The existence of the bright red supergiants in the LMC corresponding to stars up to $\sim 50 M_{\odot}$ (Humphreys and Davidson 1978) is consistent with the above scenario.

Saio, Kato, and Nomoto (1988) recently examined under what conditions a massive star undergoes a blue-red-blue evolution. The evolution of a star of initial mass $20 M_{\odot}$ star in the HR diagram is shown in Figure 1 from the zero-age main-sequence through carbon ignition at the center. The metallicity in the envelope was assumed to be $Z=0.005$ and the Schwarzschild criterion was adopted. The star shows the three types of evolutionary path (A, B, C) depending on the mass loss, metallicity, and the change in the helium abundance $Y$ in the envelope.

Such a behavior can be understood from the fact that the blue- supergiant envelope solution with only a certain combination of $\left(M_{\text {env }}, Y\right)$ fits to the evolved core model of $\sim 6 M_{\odot}$ for a given luminosity (Fig. 2a). For example, for $L=1 \times 10^{5} L_{\odot}$ and $Y=0.25$, only two solutions with $M_{\text {env }}$ $\sim 14 M_{\odot}$ (almost no mass loss) and $0.05 M_{\odot}$ are allowed to exist (see also Barkat and Wheeler 1987, Wheeler et al. 1988).

Among the three cases of A, B, and C in Fig. 1, the progenitor of SN 1987A must have evolved along the path $\mathrm{C}$ as follows.

Evolution from the Blue to the Red: The progenitor was $\sim 20 M_{\odot}$ on its main-sequence. During early helium burning, the star was a blue supergiant. Whether the star remains blue or moves to the red depends on mass loss. If $M_{\mathrm{env}}$ would be sufficiently large (i.e., almost no mass loss) for $Y \sim 0.25$ (Fig. 2a), the star would have remained blue without undergoing extensive redward evolution. (This corresponds to the models by Brunish and Truran 1982, Hillebrandt et al. 1987, and Arnett 1987.) However, the progenitor of SN 1987A should have undergone mass loss and significantly reduced its $M_{\text {env }}$. Then the star moved redward to become a red supergiant. This is because the blue supergiant envelope solution with the reduced $M_{\text {env }}$ does not exist for its $L$ and $Y$. Thus the mass loss is the driving mechanism of the redward evolution. Before reaching 


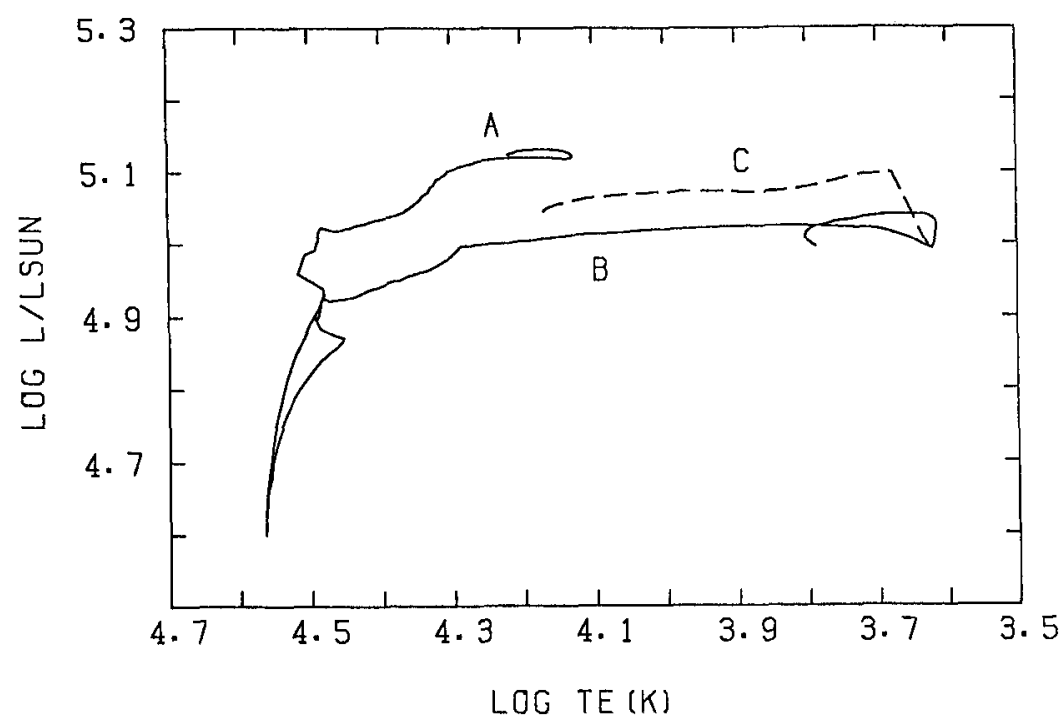

Figure 1: Evolutionary tracks in the HR diagram of the initially $20 M_{\odot}$ star. Cases A, $\mathrm{B}$, and $\mathrm{C}$ correspond to models with no mass loss (A), with no artificial enhancement of helium in the hydrogen-rich envelope (C), and with enhancement of helium up to $Y$ $=0.4(\mathrm{~B})$.
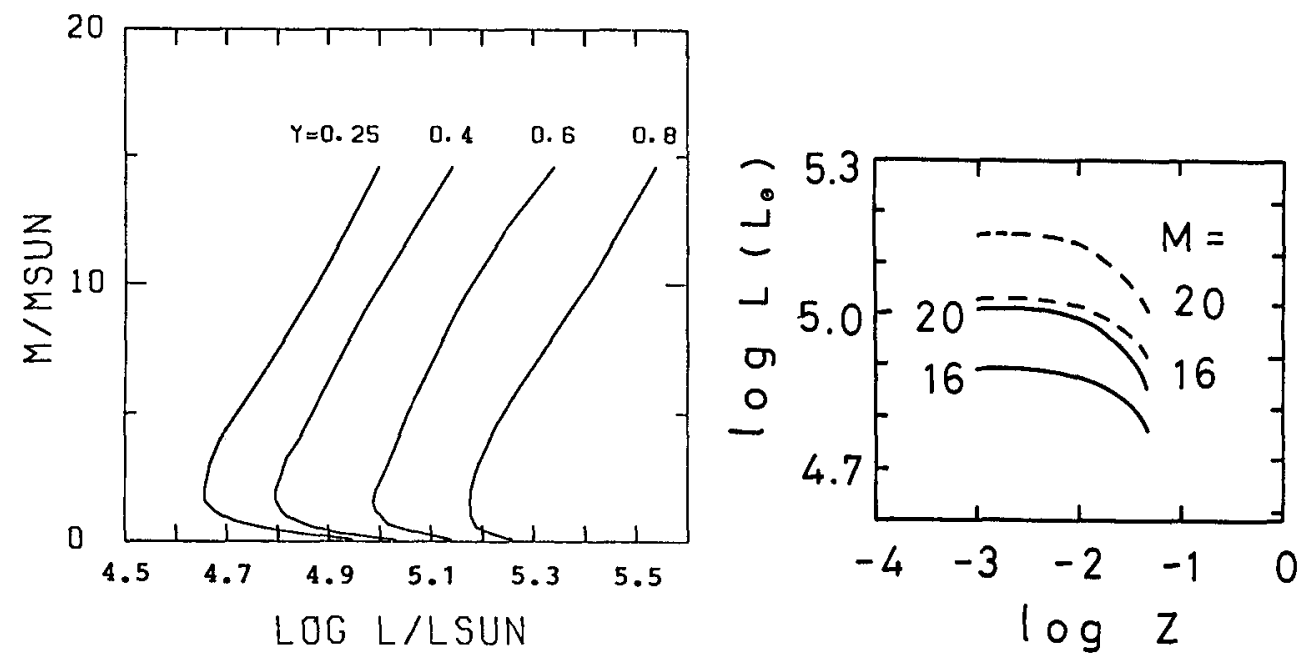

Figure 2a: Envelope solutions with the outer boundary of $\log T_{\text {eff }}=4.2$ and the inner boundary conditions that fit to the core at $M_{r}=5.45 M_{\odot}$ and $r=0.6 R_{\odot}$. The ordinate is the mass of the hydrogen-rich envelope $\left(M_{\text {env }}=M-5.45 M_{\odot}\right)$ given as a function of luminosity $L$ and the mass fraction of helium $Y$.

Figure 2b: Envelope solutions for different metallicity. For $\log T_{\text {eff }}=4.2$, the lines for the constant total mass $\left(M=M_{\text {env }}+5.45 M_{\odot}=20\right.$ and $\left.16 M_{\odot}\right)$ are given as a function of the luminosity $L$ and the mass fraction of heavy elements $Z$. The solid and dashed lines are for helium abundance $Y=0.255-Z$ and $0.4-Z$, respectively. 
the Hayashi line, the star lost as large as $5 M_{\odot}$.

Evolution from the Red to the Blue: During the red supergiant phase, envelope mass was still decreasing and the mean helium abundance $Y$ in the envelope increased. A blueward evolution began when $\mathrm{C}+\mathrm{O}$ core contracted and helium shell burning became active. Whether the star returns to the blue supergiant depends on $M_{\text {env }}$ and $Y$. If $M_{\text {env }}$ and $Y$ would not match the blue supergiant envelope solution in Fig. 2a, the star would not move blueward in the HR diagram as case $\mathrm{B}$ in Fig. 1 ; for case $\mathrm{B}, Y$ is too small for $M_{\mathrm{env}} \sim 9 M_{\odot}$. For case $\mathrm{C}$, on the contrary, the star moves to the blue because $Y$ is artificially enhanced to 0.4 . In the progenitor of SN 1987A, such an enhancement of $Y$ have occurred probably due to mixing of helium and nitrogen-rich material from the deepest layer of the envelope to the surface by the meridional circulation. (The surface convection zone may not be deep enough.) Such a material mixing drove the progenitor of SN 1987A to move to the blue just before the explosion.

Another important point for case $\mathrm{C}$ evolution is that $M_{\text {env }}$ is as large as $10 M_{\odot}$ at the supernova explosion. This is quite distinct from the solutions obtained by Maeder (1987) and Wood and Faulkner (1987) where $M_{\text {env }} \ll 0.1 M_{\odot}$. Their solutions and case C solution correspond to the small and large $M_{\text {env }}$ branches in Fig. 2a, respectively.

If the above interpretation is correct, the star with only a narrow range of $\left(M_{\mathrm{env}}, Y\right)$ can become blue and end up its life as a blue supergiant. It is interesting to note that the envelope solution depends on its metallicity $Z$ as seen in Fig. 2b. For a given $L$, the solution with larger $Z$ has a larger $M_{\text {env }}$ or larger $Y$ to compensate the larger opacity with larger $Z$. In other words, more enhancement of $Y$ is required for a given $M_{\text {env }}$ if $Z$ is larger. Therefore, the evolution from the red to the blue is more likely to occur for smaller $Z$.

\section{HYDRODYNAMICAL MODELS}

After returning from the red, the progenitor evolved to form an onion-skin like composition structure (see Nomoto and Hashimoto 1987, 1988). The hydrogen-depleted core was about $6 M_{\odot}$. The star then collapsed, made a bounce, and generated a strong shock wave either in a prompt or delayed manner. Unfortunately, the statistics of the observed neutrinos is not good enough to determine which mechanism (prompt shock or delayed neutrino heating) worked for SN 1987A and where is the mass cut that divides the neutron star and the ejecta. In the hydrodynamical calculation of the supernova explosion, therefore, the mass cut is assumed to be $1.4 M_{\odot}$ and an energy $E$ is deposited instantaneously there to generate a strong shock wave. Thus the initial model consisted of an $1.4 M_{\odot}$ point mass neutron star, heavy element layer of $2.4 M_{\odot}$, helium-rich layer of $2.2 M_{\odot}$, and the hydrogen-rich envelope of $M_{\text {env }}$. The total mass of the ejecta is $M=4.6$ $M_{\odot}+M_{\text {env }}$.

The subsequent propagation of the shock wave, the expansion of the star, and the optical light curve are calculated for models 11E1Y4, 11E1Y6, 11E1.5, 11E2, 7E1Y4, where 11 and 7 denote the ejecta mass of $M=11.3 M_{\odot}\left(M_{\mathrm{env}}=6.7 M_{\odot}\right)$ and $7.0 M_{\odot}\left(M_{\mathrm{env}}=2.4 M_{\odot}\right)$, respectively; $\mathrm{E} 1, \mathrm{E} 1.5$, and E2 denote explosion energy of $E=1,1.5$, and $2 \times 10^{51}$ erg, respectively; $\mathrm{Y} 4$ and Y6 stand for a surface composition of $Y=0.40$ and 0.6 , respectively. For all models the initial radius of the progenitor is assumed to be $R_{0}=3 \times 10^{12} \mathrm{~cm}$ (see Nomoto et al. 1987, Shigeyama et al. 1988 for details). 


\subsection{Explosive Nucleosynthesis}

As the shock wave propagates through $\mathrm{Si}$ and O-rich layers, explosive nucleosynthesis takes place behind the shock. The peak temperature at the deep layer with $M_{r}<1.67 M_{\odot}$ exceeds 5.5 $\times 10^{9} \mathrm{~K}$ so that the materials, originally composed of $\mathrm{Si}, \mathrm{S}$ and $\mathrm{Ca}$, are burned into almost nuclear statistical equilibrium (NSE) elements. Because of low density and high temperature, such a layer undergoes alpha- rich freezeout being composed of mostly ${ }^{56} \mathrm{Ni}$ and ${ }^{4} \mathrm{He}$ (Thielemann et al. 1986). In the inner O-rich layer, oxygen is burned into mostly $\mathrm{Si}, \mathrm{S}, \mathrm{Ca}$, and some ${ }^{56} \mathrm{Ni}$. The resulting composition structure after the shock passage is shown in Figure 3. The masses of ${ }^{56} \mathrm{Ni}$ produced by silicon burning at $M_{r}=1.60-1.67 M_{\odot}$ and by oxygen burning at $M_{r}>1.67 M_{\odot}$ are $0.05 M_{\odot}$ and $0.025 M_{\odot}$, respectively (Hashimoto et al. 1988).

As shown in $\S 4$, the total amount of ${ }^{56} \mathrm{Ni}$ that power the optical light curve of SN 1987A is $\sim 0.07 M_{\odot}$. This is consistent with the above composition structure if the mass cut is $1.60 M_{\odot}$. The resulting neutron star has a gravitational mass of $\sim 1.45 M_{\odot}$, which is consistent with the observations of neutrinos (e.g., Totsuka 1988; Wilson 1988).

\subsection{Shock Propagation and Material Mixing}

The shock wave propagates through the hydrogen-rich envelope. When the expansion of the inner core is decelerated by the low-density envelope, a reverse shock forms and produces a density inversion (Nomoto et al. 1987). Because of Rayleigh-Taylor instability, the core material will be mixed during early stages (see also Woosley et al. 1988). Since the time scale and the extent of mixing are uncertain, we calculated several models 11E1Y6 assuming mixing up to $M_{r} / M_{\odot}=$ 0.07 (no mixing), 3.4, 4.6 (outer edge of helium layer), and 6.0. For the mixed layer, we assumed uniform composition. Rayleigh-Taylor instability may also form clumpy medium and could cause a leak of $\gamma$-ray and $\mathrm{X}$-rays.

The shock wave arrives at the original surface of the star at $t_{\text {prop }}$, which is approximated for different values of the initial radius $R_{0}$ and the ejected mass $M$, and for different explosion energies $E$ as (Shigeyama et al. 1987, 1988)

$$
t_{\text {prop }} \sim 2 \mathrm{hr}\left(R_{0} / 3 \times 10^{12} \mathrm{~cm}\right)\left(\frac{M / 10 M_{\odot}}{E / 1 \times 10^{51} \mathrm{erg}}\right)^{1 / 2}
$$

The condition $t_{\text {prop }}<3 \mathrm{hr}$ (Kamiokande time is used) rules out all the models with too large $R_{0}$ and too low $E / M$ (Shigeyama et al. 1987). A progenitor radius larger than $4.5 \times 10^{12} \mathrm{~cm}$ is ruled out for Model 11E1 simply from this condition. If $R_{0}=3.5 \times 10^{12} \mathrm{~cm}, E / M$ should be larger than $0.6 \times 10^{50} \mathrm{erg} / M_{\odot}$.

\subsection{Velocity Profile}

After the shock wave reaches the surface, the star starts to expand and soon the expansion becomes homologous. In Fig. 4, the velocity distribution for the homologous expansion is shown for 11E1 and 11E2. The velocity gradient with respect to the enclosed mass, $M_{r}$, is very steep near the surface, while it is almost flat in the helium layer and the heavy element core. This is because the core material is decelerated and forms a dense shell due to the reverse shock. The 


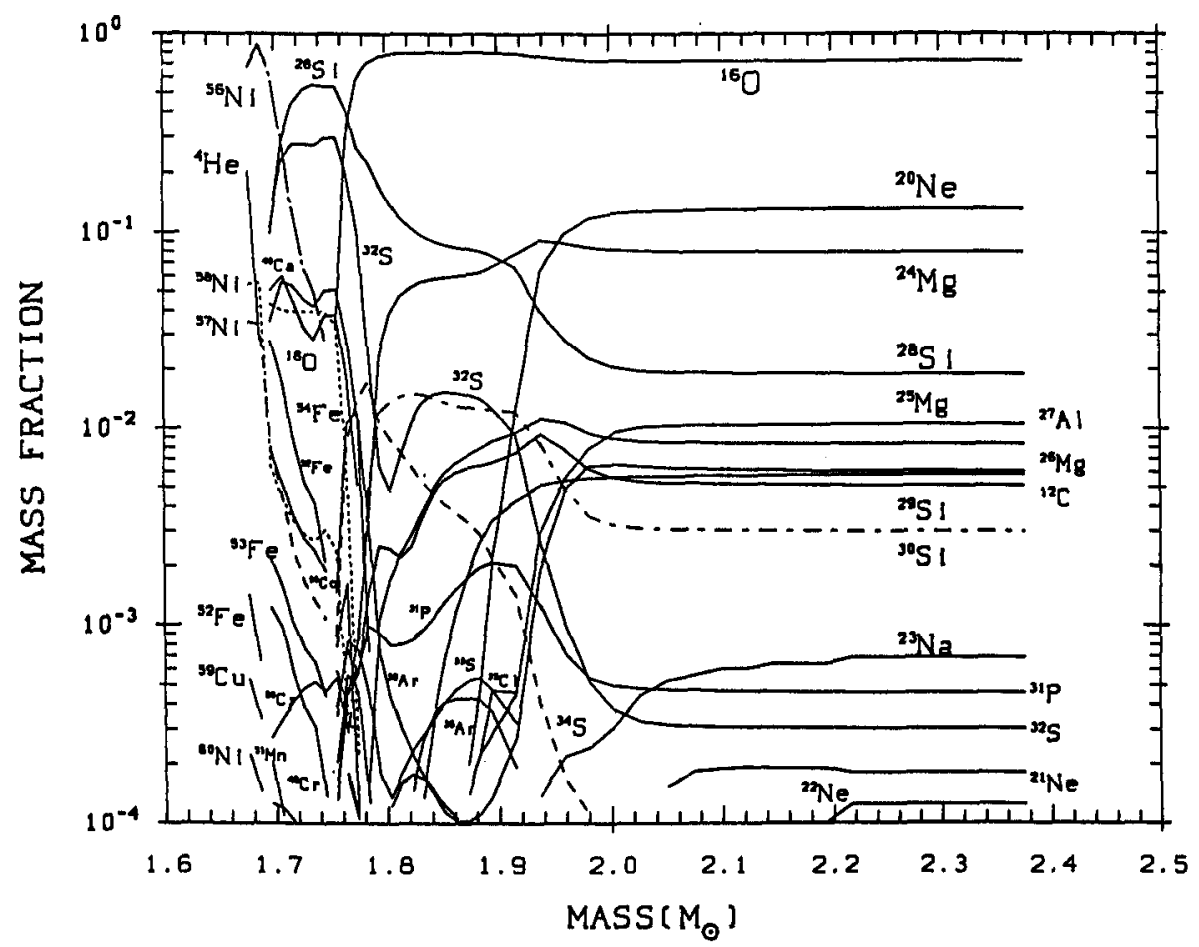

Figure 3: Abundances after explosive oxygen burning at $M_{r} \geq 1.67 M_{\odot}$ for the $6 M_{\odot}$ core.
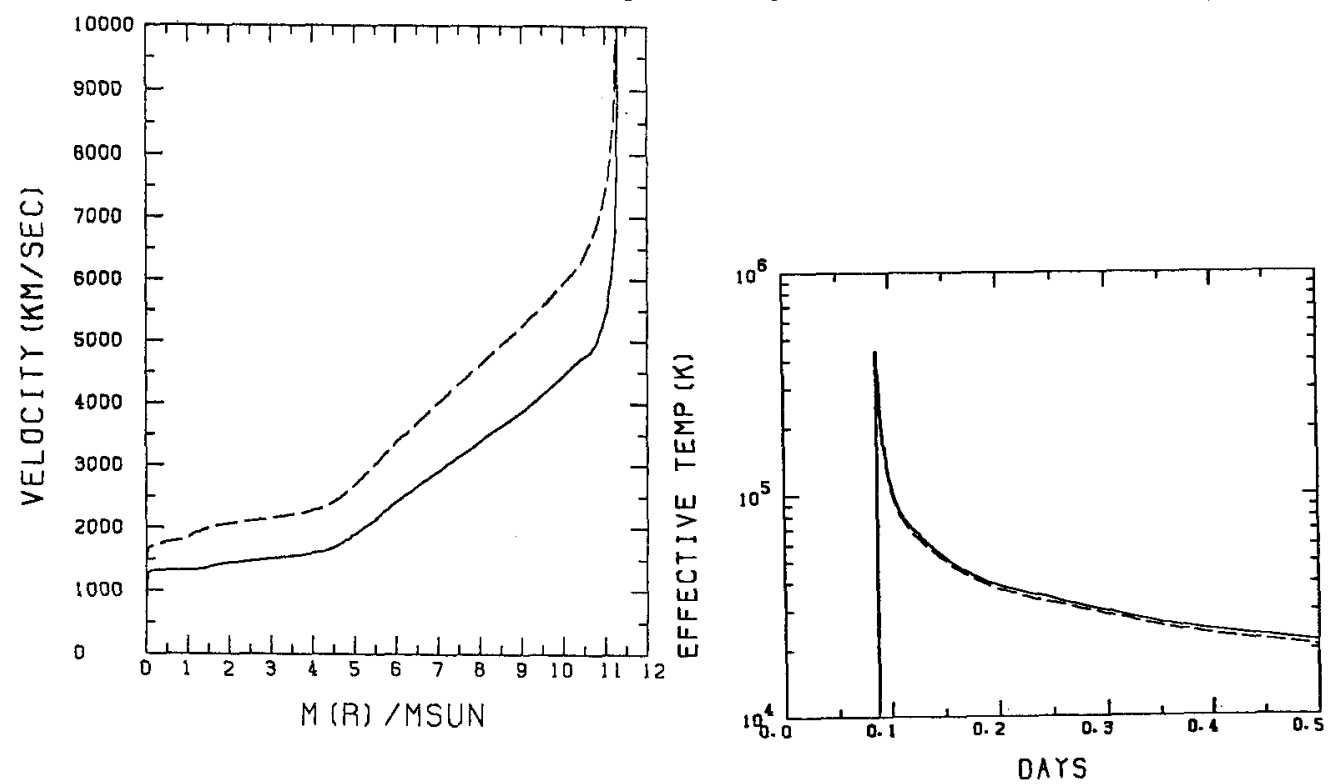

Figure 4: Velocity distribution for Models $11 \mathrm{E} 1$ (solid: $M=11.3 M_{\odot}, M_{\text {env }}=6.7$ $M_{\odot}, E=1 \times 10^{51} \mathrm{erg}$ ) and 11E2 (dashed: $E=2 \times 10^{51} \mathrm{erg}$ ) at $t=116 \mathrm{~d}$.

Figure 5: Change in the effective temperature near the shock break out at the surface for 11E1Y6 (solid) and 11E1Y4 (dashed). 
expansion velocities of the helium and heavy element layers are so small that the kinetic energy of these layers is only 10 percent of the total kinetic energy for 11E1.

Expansion velocities have been measured to give important constraints on the above hydrodynamical models (Phillips 1988). As the star expands, the photosphere moves inward in $M_{r}$. Because of the steep velocity gradient near the surface, the velocity of the material at the photosphere decreases. Good agreement between the observed and theoretical material velocities at the photosphere (Nomoto and Shigeyama 1988; Shigeyama et al. 1988) suggests $E / M_{\text {env }}$ should not be so different from $\sim 1.5 \times 10^{50} \mathrm{erg} / M_{\odot}$. The lowest velocity of the hydrogen lines would provide an information about the mixing between the hydrogen-rich envelope and the core as suggested from the optical, $\mathrm{X}$-ray, and $\boldsymbol{\gamma}$-ray light curves.

\section{LIGHT CURVE DUE TO SHOCK HEATING}

The light curve of SN 1987A shows quite unique features, which give important constraints on the hydrodynamical model of explosion. In the theoretical model, the shock wave initially establishes the radiation field with energy of roughly a half of explosion energy E; e.g., $4.4 \times 10^{50}$ erg for $11 \mathrm{E} 1$. The early light curve up to $t \sim 25 \mathrm{~d}$ can be accounted for by diffusive release of this energy.

When the shock wave arrives at the photosphere, the bolometric luminosity reaches $2 \times 10^{44}$ $\mathrm{erg} \mathrm{s}^{-1}$ and the effective temperature becomes as high as $5 \times 10^{5} \mathrm{~K}$ (Fig. 5) for Model 11E1. Hence, most of the radiation is emitted in the UV band. Total energy of radiation during the first two days amounts to $10^{47} \mathrm{erg}$, which is enough to ionize the circumstellar matter.

Afterwards, the ejected gas and the radiation field expands rapidly so that the interior temperature decreases as $r^{-1}$, i.e., almost adiabatically (Fig. 5). Most of the radiation field energy is lost by $P \mathrm{~d} V$ work. As a result of decreasing photospheric temperature, the intensity peak shifts rapidly to the optical wavelength. Two unique features of SN 1987A at this optical flare-up are: 1) It took only 3 hours for the visual magnitude to reach 6.4 magnitude after the neutrino burst (McNaught 1987). 2) At the subsequent plateau, the optical light was much dimmer than typical Type II supernovae. The steep rise in luminosity in $3 \mathrm{hr}$ and a less luminous plateau require relatively large $E / M$ and small $R_{0}$ (Shigeyama et al. 1987).

Figure 6 shows the changes in the calculated V magnitude for 11E1Y4 (dashed) and 11E1Y6 (solid). To account for the observed points, $E=1 \times 10^{51}$ erg is required for $M=11.3 M_{\odot}$. For the envelope with smaller $Y$, the luminosity is lower and hence a larger $E$ is required because of a larger scattering opacity. Moreover, the model with $Y=0.6$ is in better agreement with the overall shape of the observed light curve than $Y=0.4$ especially at $t>3 \mathrm{~d}$. Such an enhancement of helium abundance may be consistent with the restriction from the envelope solution as seen in Fig. 2a.

In the theoretical visual luminosity, there remain uncertainties. The supernova atmosphere is scattering dominated so that the color temperature may be significantly higher than the effective temperature (Shigeyama et al. 1987; Hoflich 1988), i.e., diluted black body radiation is emitted. The bolometric correction is sensitive to the color temperature because it is as high as $4-6 \times 10^{4}$ K. More careful calculations would be required to obtain accurate constraints on $R_{0}$ and $E / M$. 


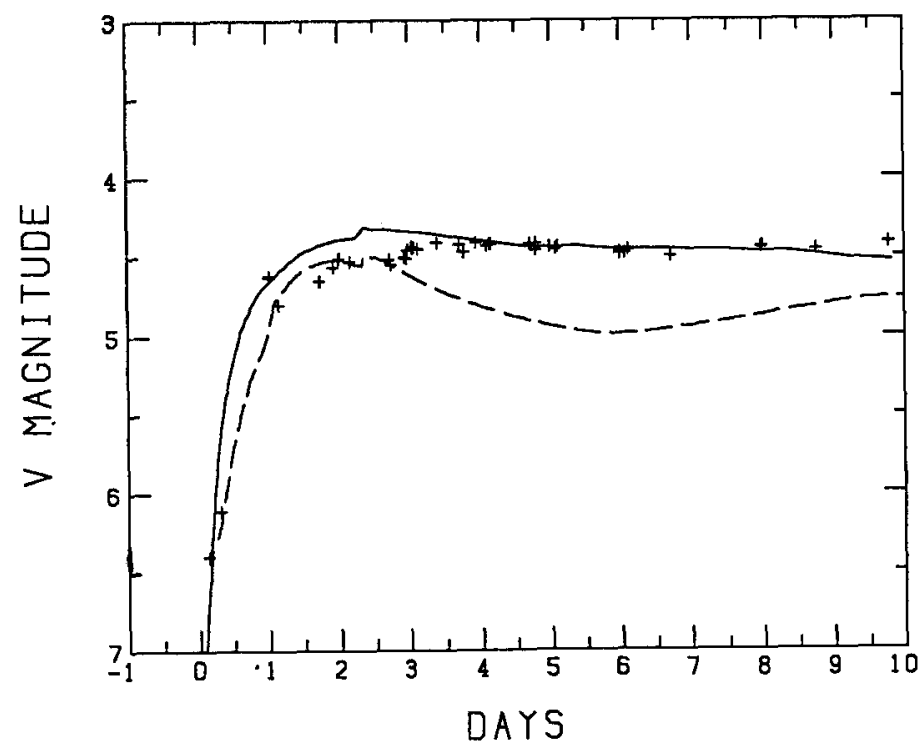

Figure 6: The calculated visual magnitude for Models 11E1Y6 (solid) and 11E1Y4 (dashed). Observed data are taken from ESO (Cristiani et al. 1987), CTIO (Blanco et al. 1987), and SAAO (Menzies et al. 1987).

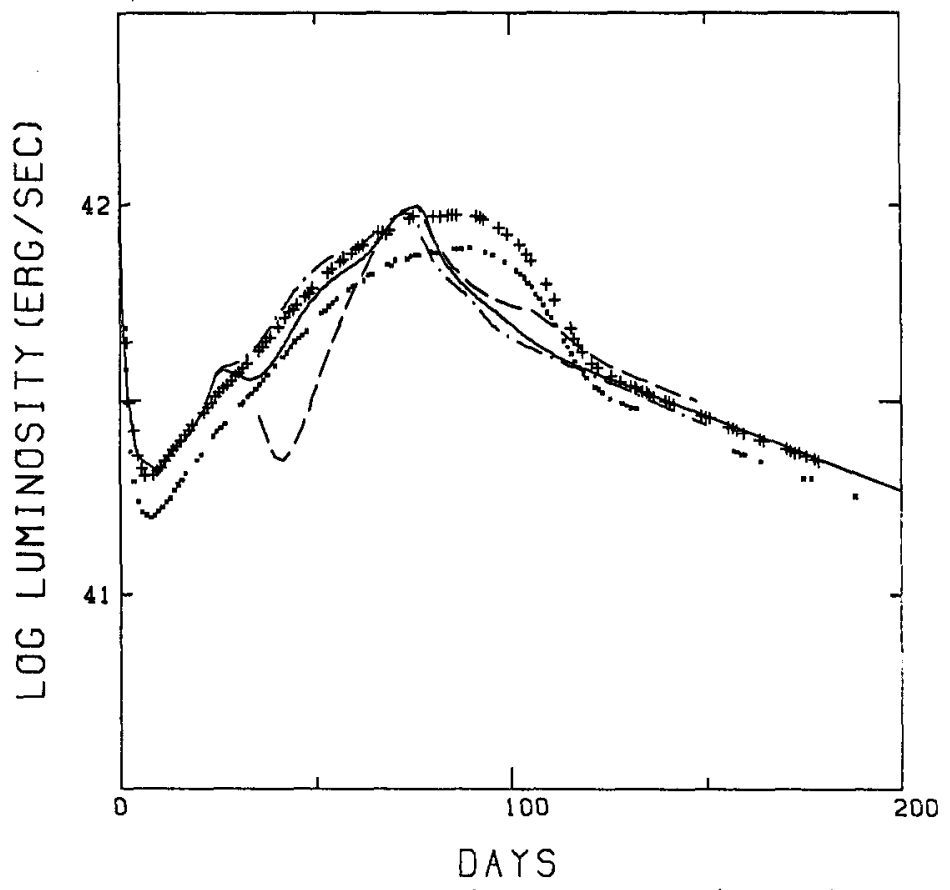

Figure 7: Bolometric light curve for several cases of mixing (11E1Y6). Production of $0.07 M_{\odot}$ ${ }^{56} \mathrm{Ni}$ is assumed. For the dashed curve ${ }^{56} \mathrm{Ni}$ is assumed to be confined in the innermost layer while the solid curve assumes that ${ }^{56} \mathrm{Ni}$ is mixed uniformly up to $M_{r}=4.6 M_{\odot}$ (solid) and $6.0 M_{\odot}$ (dash-dotted). Observed points are taken from SAAO (+; Catchpole et al. 1987) and CTIO (x; Hamuy et al. 1988). 


\section{LIGHT CURVE POWERED BY RADIOACTIVE DECAYS}

After these early stages, the observations show an increase in the bolometric luminosity. The energy source that continually heats up the expanding star is certainly the decaying ${ }^{56} \mathrm{Co}$ as evident from the light curve tail after $120 \mathrm{~d}$.

The line $\gamma$-rays from ${ }^{56} \mathrm{Co}(847 \mathrm{keV}, 1238 \mathrm{keV}, 2599 \mathrm{keV}, 1771 \mathrm{keV}, \ldots)$ undergo multiple Compton scatterings and are degraded into $\mathrm{X}$-rays. The resulting $\mathrm{X}$-rays and fast electrons ionize atoms, which recombine emitting $U V$ and optical radiation. In this way, the line $\gamma$-rays are thermalized to heat up the expanding materials until $\mathrm{X}$-rays and $\gamma$-rays emerge from the supernova due to the decrease in the column depth of the ejecta.

The theoretical light curve with radioactive decays of ${ }^{56} \mathrm{Ni}$ and ${ }^{56} \mathrm{Co}$ was calculated assuming the production of $0.07 M_{\odot}{ }^{56} \mathrm{Ni}$. Figures 7 and 8 compare the calculated bolometric light curve with observations (Catchpole et al. 1987; Hamuy et al. 1988) for several models. The light curve shape is sensitive to the hydrodynamics and thus is a useful tool to infer 1) the distribution of the heat source ${ }^{56} \mathrm{Ni}, 2$ ) the mass of the hydrogen-rich envelope $M_{\text {env }}$, and 3) the explosion energy $E$.

As the star expands, the photosphere becomes deeper as the recombination front proceeds through the hydrogen-rich envelope deeper in mass. At the same time a heat wave is propagating out from the interior. At a certain stage, energy flux due to radioactive decays exceeds that from shock heating. The dates when the radioactivity starts to dominate and when the luminosity reaches its peak depend on the above three factors as follows.

1) Mixing: Mixing of ${ }^{56} \mathrm{Ni}$ into outer layers is likely to occur due to Rayleigh-Taylor instability ( $\$ 3.2$ ). Figure 7 shows how the light curve depends on the distribution of ${ }^{56} \mathrm{Ni}$ by comparing the cases with mixing of ${ }^{56} \mathrm{Ni}$ up to $M_{r} / M_{\odot}=0.07$ (no mixing; dashed line), 4.6 (outer edge of helium layer; solid), and 6.0 (dashed-dotted) for $11 \mathrm{E} 1 \mathrm{Y} 6$. If we assume that ${ }^{56} \mathrm{Ni}$ is confined in the innermost layer of the ejecta, the increase in luminosity due to radioactive heating is delayed to $t=42 \mathrm{~d}$, a dip appears in the curve, and the light curve shape in the rising part is too steep as compared with the observations. On the other hand, if ${ }^{56} \mathrm{Ni}$ is mixed into outer layers, heat is transported to the envelope earlier. As a result the optical light increases earlier, i.e., $t=33 \mathrm{~d}$ and $26 \mathrm{~d}$ for $M_{\mathrm{mix}}=4.6$ and $6.0 M_{\odot}$, respectively, and the light curve shape is less steep, being in better agreement with the observations.

This suggests that the heat source had actually been mixed into outer layers and its effect began to dominate the light curve from $t \sim 26 \mathrm{~d}$. From the observational side, Phillips (1988) noted that the changes and kinks of the color started from $t=25 \mathrm{~d}$, which may indicate the appearance of heat flux due to radioactive decays.

2) Envelope mass: For Model 7E1 with a hydrogen-rich envelope of $2.6 M_{\odot}$, a peak luminosity of $\sim 10^{42} \mathrm{erg} \mathrm{s}^{-1}$ is reached too early at about $t=50 \mathrm{~d}$ (Fig. 8), because the expansion velocities of the helium and heavy element layers are larger than in 11E1 and the photosphere approaches the helium layer earlier. Therefore $M_{\text {env }}$ should be larger than $\sim 3 M_{\odot}$. Probably we need $M_{\text {env }}$ $=5-10 M_{\odot}$ for a good agreement with the observations.

3) Explosion Energy: For Model 11E1.5Y4 $\left(E=1.5 \times 10^{51} \mathrm{erg}\right)$, the luminosity peak of $1.3 \times 10^{42} \mathrm{erg} \mathrm{s}^{-1}$ is reached at $t=63 \mathrm{~d}$ which is a little too bright and too early compared with the observation. The cases with $E=1.0-1.3 \times 10^{51} \mathrm{erg}$ for $M_{\mathrm{env}}=6.7 M_{\odot}$ show a better fit to the observations. This constraint is consistent with the condition obtained from the photospheric velocity. 


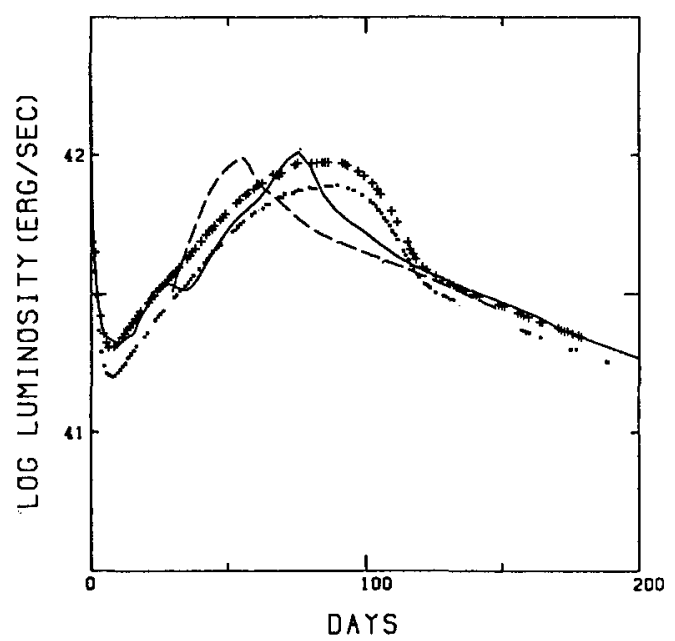

Figure 8: Dependence of the light curve on $M_{\text {env }}$. The solid line is Model 11E1Y6 ( $M$ $=11.3 M_{\odot}, M_{\text {env }}=6.7 M_{\odot}$; mixing of ${ }^{56} \mathrm{Ni}$ at $\left.M_{r}<3.4 M_{\odot}\right)$ and the dashed is 7E1Y6

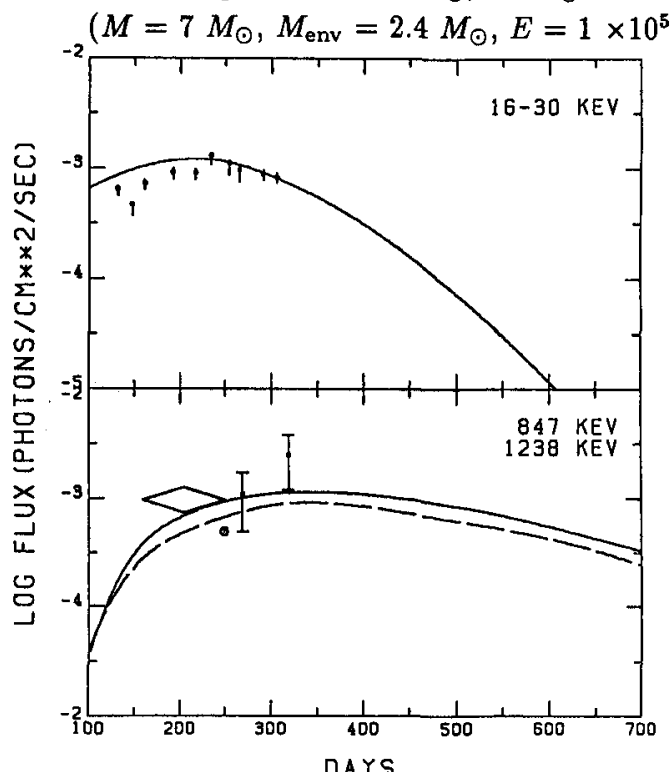
erg).

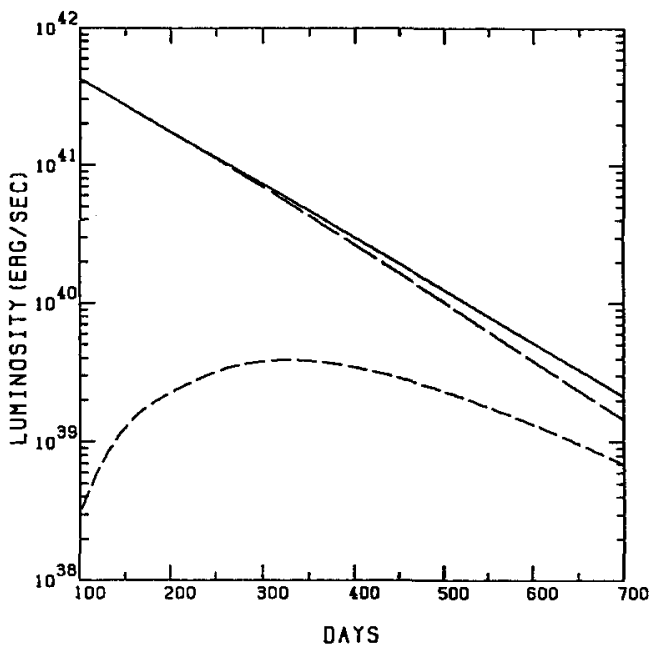

Figure 9: Calculated X-and $\gamma$-ray light curves for the hydrodynamical model 11E1Y6. $847 \mathrm{keV}$ and $1238 \mathrm{keV}$ line fluxes are shown by the solid and the dashed line, respectively. Time is measured from the beginning of explosion. ${ }^{56} \mathrm{Co}$ and other heavy elements are mixed uniformly up to the outer edge of the original helium layer $\left(M_{r}=4.6 M_{\odot}\right)$ and inhomogeneously in the hydrogenrich envelope up to $M_{r}=10 M_{\odot}$ (see text). The crosses are the X-ray light curve observed by Ginga (Makino 1988). Gamma-ray observations by SMM (Matz et al. 1988) and balloon borne observations (Sandie et al. 1988; Cook et al. 1988; Rester et al. 1988) are indicated.

Figure 10: Changes in the $\mathrm{X}$-ray and $\gamma$-ray luminosity above $5 \mathrm{keV}$ is shown by the dashed line. The associated changes in the optical bolometric luminosity (dashed) are compared with the energy generation rate due to ${ }^{56} \mathrm{Co}$ decay (solid line). 
For Model 11E1, the bolometric luminosity reaches a peak value of $L_{p k}=1 \times 10^{42}$ at $t=77$ d. After the peak, the luminosity decreases more rapidly than SN $1987 \mathrm{~A}$ which formed a broad peak. Finally the light curve enters the radioactive tail (Fig. 7).

It should be noted that the observed peak luminosity is higher than the energy generation rate due to Co-decay which gives $L=5.4 \times 10^{41} \mathrm{erg} \mathrm{s}^{-1}$ at $t=70 \mathrm{~d}$ for $M_{\mathrm{Co}}=0.07 M_{\odot}$. This implies that previously deposited energy from Co-decay is also radiated away during the broad peak of the light curve (Woosley et al. 1988). How this additional energy is radiated away is rather sensitive to the dynamical behavior and to the opacity. The difficulty to reproduce the broad peak may require the improvement of the flux-limited diffusion approximation used in the calculation.

Despite this difficulty, both the peak luminosity and the tail can be consistently accounted for by the radioactive decay of ${ }^{56} \mathrm{Co}$ whose mass is $\sim 0.07 M_{\odot}$.

\section{HARD X-RAYS AND GAMMA-RAYS}

As the column depth of the supernova ejecta decreases as $t^{-2}, \gamma$-rays and hard X-rays suffer from less Compton scattering and photoelectric absorption and eventually emerge from the surface. The timing of their emergences and light curves are sensitive to $E, M_{\text {env }}$, and the distribution of elements. In other words, $\mathrm{X}$-ray and $\gamma$-ray observations provide another good diagnosis of the supernova interior.

$\mathrm{X}$-ray and $\gamma$-ray light curves for model 11E1Y6 were calculated by Itoh et al. (1987) and Ebisuzaki and Shibazaki (1988). They have shown that ${ }^{56} \mathrm{Co}$ needs to be mixed into hydrogen-rich envelope (i.e., the column depth to the cobalt layer is $\sim 20 \mathrm{~g} \mathrm{~cm}^{-2}$ at $t=200 \mathrm{~d}$ ) to account for the emergence of the X-rays as early as observed by Ginga (Dotani et al. 1987) and Kvant (Sunyaev et al. 1987). (See also Pinto and Woosley 1988 and Sutherland et al. 1988). Recently, it is reported that SMM has been observing $\gamma$-rays since August 1987 (Matz et al. 1988). Such an early $\gamma$-ray emergence requires the column depth to the ${ }^{56} \mathrm{Co}$ layer as small as $\sim 2.5 \mathrm{~g} \mathrm{~cm}^{-2}$ at $t=200 \mathrm{~d}$ (Itoh et al. 1988; Kumagai et al. 1988). Figure 9 shows the calculated light curves of $16-30 \mathrm{keV}$ $\mathrm{X}$-ray and the $847 \mathrm{keV}$ and $1238 \mathrm{keV}$ line $\gamma$-rays. They are consistent with the observations. This model assumes a step-like distribution of ${ }^{56} \mathrm{Co}$ where the mass fraction of ${ }^{56} \mathrm{Co}$ in the layers at $M_{\text {r }}$ $\leq 4.6 M_{\odot}, 4.6-6 M_{\odot}, 6-8 M_{\odot}$, and $8-10 M_{\odot}$ are $X_{\mathrm{Co}}=0.0128,0.0035,0.0021$, and 0.0011 , respectively. The X-ray spectrum and its time evolution are in good agreement with observations (see Itoh et al. 1988 in this volume).

This model predicts:

1) The X-ray flux at $20-30 \mathrm{keV}$ will decrease below the detection limit of Ginga around $t \sim 400$ $\mathrm{d}$ while harder $\mathrm{X}$-rays remain observable until $\mathrm{t} \sim 2$ years.

2) The line $\gamma$-rays will be observable until $t \sim 2$ yr (Fig. 9).

3) With increasing high energy flux, the deviation of the optical bolometric luminosity from the exponential decline will become significant as seen in Figure 10. The luminosity above $5 \mathrm{keV}$ reaches 5 and $8 \%$ of the energy generation rate at $t=300 \mathrm{~d}$ and $350 \mathrm{~d}$, respectively.

Further satellite and balloon-borne observations will test the predicted spectral evolution and light curves and provide new information on the distribution of the mixed heavy elements in the supernova ejecta. 


\section{LIGHT CURVE MODELS FOR TYPE Ib SUPERNOVAE}

Now that some details of SN 1987A has been analyzed, the relation between SN 1987A and Type Ib supernovae (SN Ib) is worth studying. Massive helium stars have been the most popular progenitor model for Type Ib supernovae (e.g., Wheeler et al. 1987), while a helium detonation in accreting white dwarfs could be a possible alternative model (e.g., Branch and Nomoto 1986). If massive helium stars are the SN Ib progenitor, there would be a similarity in the core structure, explosion energy, and nucleosynthesis between SN 1987A and SN Ib. Then the same parameter could reproduce the light curve of SN Ib because of the difference in the envelope structure.

By applying the same physics to the same $6 M_{\odot}$ helium core model as used in the modeling of SN $1987 \mathrm{~A}$, light curves powered by the decay of $0.07 M_{\odot}{ }^{56} \mathrm{Co}$ are calculated and compared with observations. The $\gamma$-ray opacity of $0.03 \mathrm{~cm}^{2} \mathrm{~g}^{-1}$ (Sutherland and Wheeler 1984) and the optical opacity of $0.1 \mathrm{~cm}^{2} \mathrm{~g}^{-1}$ are assumed. For the $6 M_{\odot}$ helium star with $E=1 \times 10^{51} \mathrm{erg}$ (where the masses of the ejecta $M_{\mathrm{ej}}$ and the neutron star residue are assumed to be $4.4 M_{\odot}$ and 1.6 $M_{\odot}$, respectively), the calculated bolometric light curve after the peak declines at nearly the ${ }^{56} \mathrm{Co}$ decay rate because almost all $\gamma$-ray energies are deposited in the star (see also Schaeffer et al. 1987; Ensman and Woosley 1988). This is slower than even the slowest SN Ib, SN 1984L (Harkness et al. 1987).

The decline rate of the light curve depends largely on how fast $\gamma$-rays and X-rays escape from the star. The column depth to the cobalt layer at time $t$ is roughly proportional to (mass of the overlying layer $)^{2} /\left(E t^{2}\right)$ (e.g., Chan and Lingenfelter 1987). Accordingly the optical light curve declines faster, if the ejecta mass is smaller, ${ }^{56} \mathrm{Co}$ is mixed closer to the surface, and $E$ is larger.

Possibilities that $M_{\mathrm{ej}}$ is smaller than $4.4 M_{\odot}$ include: 1 ) the helium star itself is smaller than $6 M_{\odot}$, e.g., $4-5 M_{\odot}$, and 2) the helium star undergoes mass loss and loses most of its helium envelope before the explosion. To explore the most extreme case of mass loss, we removed the helium envelope to expose the $\mathrm{C}+\mathrm{O}$ layer. Then $M_{\mathrm{ej}}=2.2 M_{\odot}$. (This also corresponds to the less massive helium star of $3.6-3.8 M_{\odot}$.) Its light curve is shown by the dashed curve in Fig. 11 and is consistent with SN 1984L. However, it is still slower than the bolometric light curve of SN 1983N (Panagia 1987) and the photographic light curve of SN 1964L (Bertola et al. 1965).

The other possibility is the mixing of ${ }^{56} \mathrm{Co}$ into outer layers. The solid line in Fig. 11 shows a light curve for rather extreme case of mixing where ${ }^{56} \mathrm{Co}$ is uniformly distributed in the whole

- ejecta of 2.2 $M_{\odot}$. The light curve near the peak is consistent with SN $1983 \mathrm{~N}$ but its decline after $\sim 35 \mathrm{~d}$ is still slower than the observations (see also Ensman and Woosley 1988). Even for $E=3$ $\times 10^{51}$ erg with uniform mixing of ${ }^{56} \mathrm{Ni}$, the decline shown by the dashed curve in Fig. 12 is slower than SN 1983N. Since the column density of the ejecta scales as $M_{\mathrm{ej}}{ }^{2} / E t^{2}$, the dashed curve in Fig. 12 suggests $M_{\mathrm{ej}}$ is as small as the white dwarf mass if $E \sim 1 \times 10^{51} \mathrm{erg}$.

Finally, we tried a numerical experiment where ${ }^{56} \mathrm{Ni}$ exists only in the outermost $0.2 M_{\odot}$ layer beneath the surface. The escape of X-rays and $\gamma$-rays are significant and the resulting optical light curve (the dash-dotted curve in Fig. 11) is as narrow as SN 1964L. Such a composition inversion is not realistic for helium stars but a natural outcome from the off-center detonation in accreting white dwarfs.

The above light curve modeling is meant to explore rather extreme cases and quite preliminary, but suggestive.

1) The slowest light curve of SN Ib such as SN 1984L may be accounted for by the helium star 


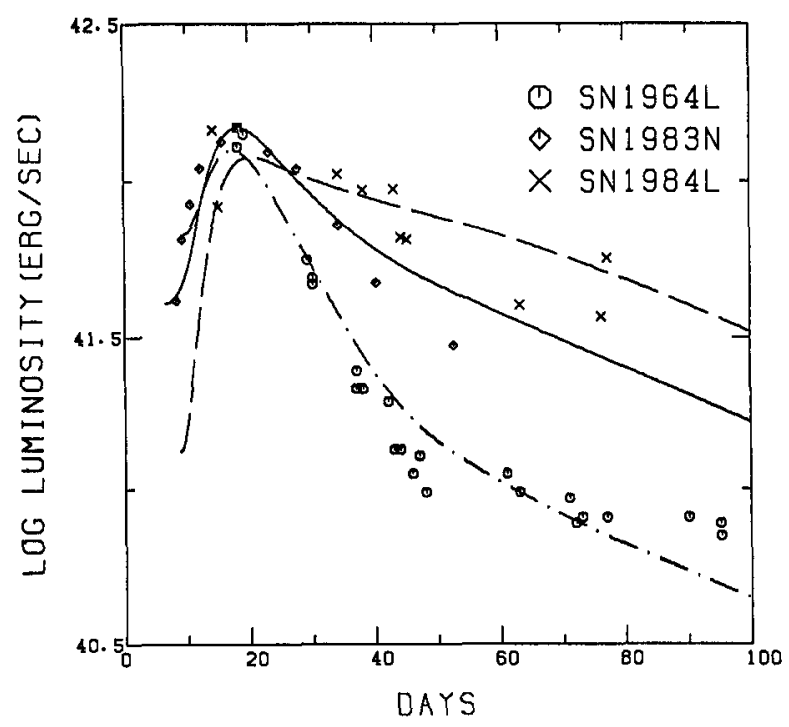

Figure 11: Calculated bolometric light curves for the ejecta of $2.2 M_{\odot}$ and $E=1$ $\times 10^{51} \mathrm{erg}$. The mass of ${ }^{56} \mathrm{Ni}$ and the optical opacity are assumed to be $0.07 M_{\odot}$ and $0.1 \mathrm{~cm}^{2} \mathrm{~g}^{-1}$, respectively. Shown are the cases where ${ }^{56} \mathrm{Ni}$ is confined in the central region (dashed), uniformly distributed throughout the ejecta (solid), and confined in the outer layers of $0.2 M_{\odot}$ below the surface (dash-dotted). The observed light curve of $1983 \mathrm{~N}$ is bolometric, while those of $1984 \mathrm{~L}$ and $1964 \mathrm{~L}$ are visual and photographic, respectively.

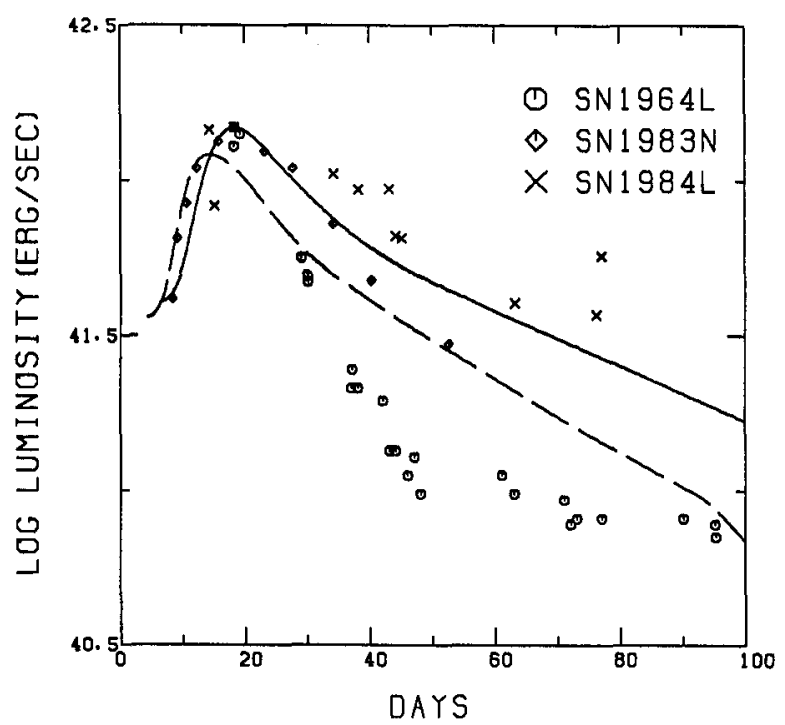

Figure 12: Same as FIg. 11 but for different explosion energy $E=1 \times 10^{51} \mathrm{erg}$ (solid) and $3 \times 10^{51} \mathrm{erg}$ (dashed). ${ }^{56} \mathrm{Ni}$ is uniformly distributed in the ejecta of $2.2 M_{\odot}$. 
models of $M_{\mathrm{ej}} \sim 2-3 M_{\odot}$ even if ${ }^{56} \mathrm{Ni}$ is confined in the central region. The origin of such a helium star is likely to be a mass exchange in the interacting binary.

2) The light curve of SN $1983 \mathrm{~N}$ may indicate that $M_{\mathrm{ej}}$ is as small as white dwarf mass and a large scale mixing in the ejecta occurs to bring ${ }^{56} \mathrm{Ni}$ into fairly outer layers. Such a mixing of radioactive materials into outer layers might be an origin of a large non-LTE excitation of helium lines (Branch 1988).

3) If the width of the bolometric light curve of SN 1964L is even narrower than SN $1983 \mathrm{~N}$ as is the case for the blue magnitude, SN 1964L could be essentially different from SN 1983N and 1984L and belongs to a separate class of SN I (i.e., SN Ic). The progenitor could be an accreting white dwarf having some composition inversion. It is interesting that the spectra of SN 1964L and 1962L are similar to SN $1983 \mathrm{I}$ and $1983 \mathrm{~V}$ for which helium lines are weak (Wheeler et al. 1987). Also the expansion velocity at the photosphere of $1983 \mathrm{~V}$ observed near maximum light is as high as 18,000 $\mathrm{km} \mathrm{s}^{-1}$ (Branch 1988).

To clarify whether a separate class of SN Ic exists, observations and modeling of nebula phase spectra of SN Ic would be crucial. Also the mechanism of extensive mass loss and mixing required from the helium star model need to be studied.

\section{CONCLUDING REMARKS}

Comparison between the presupernova evolutionary model, hydrodynamical models and the observations imposes several interesting constraints on $M_{\text {env }}$ and $E$, i.e., on the presupernova mass loss history, the explosion mechanism, and material mixing before and after the explosion. These are summarized as follows:

1) The optical light curve at very early phase and near maximum gives a lower bound and an upper bound of $E / M_{\mathrm{env}}$, respectively. Reasonable agreement between the model and observations is obtained for $E / M_{\mathrm{env}}=1.5 \pm 0.5 \times 10^{50} \mathrm{erg} / M_{\odot}$ (see also Woosley 1988 ).

2) The near maximum light curve sets a lower bound of $M_{\text {env }}$ which is about $3 M_{\odot} \cdot M_{\text {env }} \sim 5$ - $10 M_{\odot}$ would be more reasonable. Kirshner (1988) and Dopita (1988) have also estimated that $M_{\text {env }}$ is fairly large.

3) Another constraint on $M_{\text {env }}$ is obtained from the presupernova evolutionary model where the star evolves from the blue to the red when the mass of the hydrogen-rich envelope significantly decreases and comes back from the red to the blue if the helium abundance is sufficiently enhanced by mass loss and mixing (Saio, Kato, and Nomoto 1988). The observed N/C ratio, which is $\sim 40$ times the solar ratio (Panagia 1988), is consistent with $M_{\text {env }} \sim 7-11 M_{\odot}$.

Enhancement of helium and nitrogen may require mixing due to meridional circulation because the convection in the current model is too shallow. The observed enhancement of s-process elements, $\mathrm{Sr}, \mathrm{Ba}$ (Williams 1988) might be related to this mixing.

4) Mixing of ${ }^{56} \mathrm{Co}$ into hydrogen-rich envelope may be required to account for the optical light curve and the early emergence of X-rays and $\gamma$-rays. Mechanism of mixing needs to be explored.

We would like to thank Drs. R. Catchpole, J. Danziger, M. Dopita, R. Kirshner, and R. Williams for sending us data taken at SAAO, ESO, MSSSO, IUE, and CTIO, respectively, prior to publication. A part of this work is based on the collaboration with Drs. H. Saio, M. Kato, M. Itoh, and Ms. S. Kumagai. We are grateful to them for their contribution. This work has 
been supported in part by the Grant-in-Aid for Scientific Research (62540183) of the Ministry of Education, Science, and Culture in Japan and by the Space Data Analysis Center, Institute of Space and Astronautical Sciences.

\section{References}

Arnett, W.D.: 1987, Astrophys. J., 319, 136.

Barkat, Z., and Wheeler, J.C. 1988, Astrophys. J., in press.

Bertola, F., Mammano, A., Perinotto, M.: 1965, Contr. Asiago Obs., No. 174.

Blanco, V.M., et al.: 1987, Astrophys. J., 320, 589

Branch, D.: 1988, in IAU Colloquium 108, Atmospheric Diagnostics of Stellar Evolution, ed.

K. Nomoto, Springer-Verlag, p. 281.

Branch, D., and Nomoto, K.: 1986, Astron. Astrophys., 164, L13.

Brunish, W.M. and Truran, J.W. 1982, Astrophys. J. Suppl., 49, 447.

Catchpole, R. et al.: 1987, Monthly Notice Roy. Astron. Soc., 229, 15p.

Chalabaev, A., Perrier, C., and Mariotti, J.M. 1987, IAU Circular 4481.

Chan, K.W., Lingenfelter, R.E.:1987, Astrophys. J. Letters, 318, L51.

Cook, W.R., Palmer, D., Prince, T., Schindler, C., Starr, C., Stone, E.: 1988, IAU Circular 4527.

Cristiani, S., et al.: 1987, Astron. Astrophys., 177, L5

Dopita, M.: 1988, Nature, 331, 506.

Dotani, T. et al.: 1987, Nature, 330, 230.

Ebisuzaki, T., Shibazaki, N.: 1988 , Astrophys. J. Letters, 327

Ensman, L., Woosley, S.E.: 1988, preprint.

Hamuy, M., Suntzeff, N.B., Gonzalez, R., Martin, G.: 1987, Astron. J., 95, 63.

Harkness, R.P. et al.: 1987, Astrophys. J., 317, 355.

Hashimoto, M., Nomoto, K., Shigeyama, T.: 1987, in preparation.

Hillebrandt, W., Hoflich, P., Truran, J.W., Weiss, A.: 1987, Nature, 327, 597.

Hoflich, P.: 1988, in IAU Colloquium 108, Atmospheric Diagnostics of Stellar Evolution, ed.

K. Nomoto, Springer-Verlag, p. 288.

Humphreys, R.M., Davidson, K.: 1978, Astrophys. J., 232, 409.

Itoh, M., Kumagai, S., Shigeyama, T., Nomoto, K., Nishimura, J.: 1987, Nature, 330, 233.

Itoh, M., Kumagai, S., Shigeyama, T., Nomoto, K., and Nishimura,J.: 1988, in IAU Colloquium 108, Atmospheric Diagnostics of Stellar Evolution, ed. K. Nomoto, Springer-Verlag, p. 446.

Kirshner, R.: 1988, in IAU Colloquium 108, Atmospheric Diagnostics of Stellar Evolution, ed.

K. Nomoto, Springer-Verlag, p. 252.

Kumagai, S., Itoh, M., Shigeyama, T., Nomoto, K., Nishimura, J.: 1988, in Supernova 1987A in the $\mathrm{LMC}$, ed. M. Kafatos, Cambridge University Press, in press.

Maeder, A.: 1987, in SN 1987A, ed. I.J. Danziger, ESO, Garching, p. 251.

Makino, F.: 1988, IAU Circular 4532.

Matz, S.M., Share, G.H., Leising, M.D., Chupp, E.L., Vestrand, W.T., Purcell, W.R., Strickman, M.S., Reppin, C.: 1988, Nature, 331, 416.

Menzies, J.M., et al.: 1987, Monthly Notice Roy. Astron. Soc., 227, 39

McNaught, R.H.: 1987, IAU Circ. No. 4389.

Nomoto, K., Hashimoto, M.: 1986, Prog. Part. Nucl. Phys. 17, 267. 
Nomoto, K., Hashimoto, M.: 1987, in Chemical Evolution of Galaxies with Active Star Formation, ed. K. Takakubo., Universal Acad. Press. p. 93.

Nomoto, K., Hashimoto, M.: 1988, in Theory of Supernovae, ed. G.E. Brown, Physics Report, in press.

Nomoto, K., Shigexama, T.: 1988, in Supernova 1987 A in the LMC, ed. M. Kafatos, Cambridge University Press, in press.

Nomoto, K., Shigeyama, T., Hashimoto, M.: 1987, in SN 1987A, ed. I.J. Danziger, ESO: Garching, p. 325 .

Panagia, N.: 1987, in High Energy Phenomena Around Collapsed Stars, ed. F. Pacini, D. Reidel, p. 33.

Panagia, N. et al.: 1987, IAU Circular 4514.

Phillips, M.M.: 1988, in Supernova $1987 \mathrm{~A}$ in the $L M C$, ed. M. Kafatos, Cambridge University Press, in press.

Pinto, P., Woosley, S.E.: 1988, Astrophys. J., 329, in press.

Saio, H., Kato, M., Nomoto, K.: 1988, Astrophys. J., 331, in press.

Sandie, W.G., Nakano, G.H., Chase, L.F., Jr., Fishman, G.J., Meegan, C.A., Wilson, R.B., Paciesas, W., Lashe, G.P.: 1988, preprint.

Schaeffer, R., Casse, M., Cahen, S.: 1987, Astrophys. J., 316, L31.

Shigeyama, T., Nomoto, K., Hashimoto, M.: 1988, Astron. Astrophys., in press.

Shigeyama, T., Nomoto, K., Hashimoto, M., Sugimoto, D.: 1987, Nature, 328, 320.

Sunyaev, R. et al.: 1987, Nature, 330, 227.

Sutherland, P., Xu, Y., McCray, R., Ross, R.P.: 1988, in IAU Colloquium 108, Atmospheric Diagnostics of Stellar Evolution, ed. K. Nomoto, Springer-Verlag, p. 394.

Sutherland, P., Wheeler, J.C.: 1984, Astrophys. J., 280, 282.

Thielemann, F.-K., Nomoto, K., and Yokoi, K.: 1986, Astron. Astrophys., 158, 17.

Totsuka, Y.: 1988, in IAU Colloquium 108, Atmospheric Diagnostics of Stellar Evolution, ed. K. Nomoto, Springer-Verlag, p. 335.

Wheeler, J.C., Harkness, R.P., Barker, E.S., Cochran, A.L., Wills, D.: 1987, Astrophys. J. Letters, 313, L69.

Wheeler, J.C., Harkness, R.P., Barkat, Z.: 1988, in Supernova 1987A in the LMC, ed. M. Kafatos, Cambridge University Press, in press.

Williams, R.: 1988, in IAU Colloquium 108, Atmospheric Diagnostics of Stellar Evolution, ed. K. Nomoto, Springer-Verlag, p. 274.

Wilson, R.B., Fishman, G., Meegan, C., Paciesas, W., Sandie, W., Chase, L., Nakano, G.: 1988, Nature, submitted.

Wilson, J.R.: 1988, in IAU Colloquium 108, Atmospheric Diagnostics of Stellar Evolution, ed.

K. Nomoto, Springer-Verlag, p. 348.

Wood, P.R., Faulkner, D.J.: 1988, in IAU Colloquium 108, Atmospheric Diagnostics of Stellar Evolution, ed. K. Nomoto, Springer-Verlag, p. 410.

Woosley, S.E.: 1988, Astrophys. J., in press.

Woosley, S.E., Pinto, P., Ensman, L.: 1988, Astrophys. J., 324, 466.

Zoltowski, F.: 1987, IAU Circular 4389. 